\title{
TEMPLATES FOR THE $k$-BINOMIAL COMPLEXITY OF THE TRIBONACCI WORD
}

\author{
MARIE LEJEUNE, MICHEL RIGO, AND MATTHIEU ROSENFELD
}

\begin{abstract}
Consider the $k$-binomial equivalence: two finite words are equivalent if they share the same subwords of length at most $k$ with the same multiplicities. With this relation, the $k$-binomial complexity of an infinite word $\mathbf{x}$ maps the integer $n$ to the number of pairwise nonequivalent factors of length $n$ occurring in $\mathbf{x}$. In this paper based on the notion of template introduced by Currie et al., we show that, for all $k \geq 2$, the $k$-binomial complexity of the Tribonacci word coincides with its usual factor complexity $p(n)=2 n+1$. A similar result was already known for Sturmian words but the proof relies on completely different techniques that seemingly could not be applied for Tribonacci.
\end{abstract}

\section{INTRODUCTION}

Abelian equivalence of words has been investigated for quite a long time, e.g., in the sixties Erdös raised the question whether abelian squares can be avoided by an infinite word over an alphabet of size $4,[6,17,16$. Let $\Sigma$ be a finite alphabet. We let $\Sigma^{*}$ denote the set of all finite words over $\Sigma$. Two words $u$ and $v$ in $\Sigma^{*}$ are abelian equivalent if and only if $|u|_{a}=|v|_{a}$, for all $a \in \Sigma$, where we let $|u|_{a}$ denote the number of occurrences of the letter $a$ in $u$, i.e., one word is obtained by permuting the letters of the other word.

Definition 1. Let $u$ be a word over an ordered alphabet $\{0, \ldots, k-1\}$. The abelianization or Parikh vector ${ }^{1}$ of $u$, denoted by $\Psi^{\prime}(u)$, is the column vector in $\mathbb{N}^{k}$

$$
\left(|u|_{0}, \ldots,|u|_{k-1}\right)^{\top} .
$$

With this notation, two words are abelian equivalent if and only if $\Psi^{\prime}(u)=$ $\Psi^{\prime}(v)$. A possible generalization of abelian equivalence is the $k$-binomial equivalence based on binomial coefficient of words. An independent generalization is the $k$-abelian equivalence where one counts factors of length at most $k$ [10, 11]. For a survey, see, for instance, [19]. We let the binomial coefficient $\left(\begin{array}{l}u \\ v\end{array}\right)$ denote the number of times $v$ appears as a (not necessarily contiguous) subsequence of $u$. Let $k \geq 1$ be an integer. Two words $u$ and $v$

\footnotetext{
The first author is supported by a FNRS fellowship.

${ }^{1}$ The term Parikh vector comes from Parikh's theorem in formal language theory stating that if $L$ is a context-free language, then $\Psi(L)$ is a semi-linear set. This terminology is now consecrated even though abelianization should be more suited in a mathematical context.
} 
are $k$-binomially equivalent, denoted $u \sim_{k} v$, if $\left(\begin{array}{l}u \\ x\end{array}\right)=\left(\begin{array}{l}v \\ x\end{array}\right)$ for all words $x$ of length at most $k$.

Definition 2. Let $\mathbf{x}$ be an infinite word. The $k$-binomial complexity function of $\mathbf{x}$ is defined as

$$
b_{\mathbf{x}, k}: \mathbb{N} \rightarrow \mathbb{N}, n \mapsto \#\left(F a c_{n}(\mathbf{x}) / \sim_{k}\right)
$$

where $F_{a c}(\mathbf{x})$ is the set of factors of length $n$ occurring in $\mathbf{x}$. For $k=1$, this measure of complexity is exactly the abelian complexity.

The celebrated theorem of Morse-Hedlund characterizes ultimately periodic words in terms of a bounded factor complexity function, for a reference, see [3] or [4, Section 4.3]. Hence, aperiodic words with the lowest factor complexity are exactly the Sturmian words characterized by $p_{\mathbf{x}}(n)=n+1$. It is also a well-known result of Cobham that the factor complexity of any $k$ automatic sequence is in $\mathcal{O}(n)$. The Tribonacci word has a factor complexity $2 n+1$.

We collect the known facts about the $k$-binomial complexity. For all $k \geq 2$, Sturmian words have a $k$-binomial complexity which is the same as their factor complexity, i.e., $b_{\mathbf{x}, k}(n)=n+1$ for all $n$. Since $b_{\mathbf{x}, k}(n) \leq b_{\mathbf{x}, k+1}(n)$, the proof consists in showing that any two distinct factors of length $n$ occurring in a given Sturmian word are never 2-binomially equivalent [17, Thm. 7]. However, the Thue-Morse word has a bounded $k$-binomial complexity [17, Thm. 13]. So we have a striking difference with the most usual complexity measures. Naturally, the bound on the $k$-binomial complexity of the ThueMorse word depends on the parameter $k$ because when $k$ tends to infinity, the $k$-binomial equivalence gets closer to equality of factors, i.e. $b_{\mathbf{x}, k}(n)=p_{\mathbf{x}, k}(n)$ for all $n \leq k$, and the Thue-Morse word has a factor complexity in $\Theta(n)$. The precise results are recalled below.

Theorem 3. 17] Let $k \geq 1$. There exists $C_{k}>0$ such that the $k$-binomial complexity of the Thue-Morse word $\mathbf{t}$ satisfies $b_{\mathbf{t}, k}(n) \leq C_{k}$ for all $n \geq 0$.

Theorem 4. 12] We let $\mathbf{t}$ denote the Thue-Morse word over a 2-letter alphabet. Let $k$ be a positive integer. For all $n \leq 2^{k}-1$, we have $b_{\mathbf{t}, k}(n)=$ $p_{\mathbf{t}}(n)$. For all $n \geq 2^{k}$, we have

$$
b_{\mathbf{t}, k}(n)=\left\{\begin{array}{l}
3 \cdot 2^{k}-3, \text { if } n \equiv 0 \quad \bmod 2^{k} \\
3 \cdot 2^{k}-4, \text { otherwise. }
\end{array}\right.
$$

When investigating this new $k$-binomial complexity measure, it is naturally interesting to consider various well-known words or families of words. A natural choice is therefore to try computing the $k$-binomial complexity of the Tribonacci word $010201001020101 \cdots$, fixed point of the morphism $\tau: 0 \mapsto 01,1 \mapsto 02,2 \mapsto 0$. From computer experiments, the second author made the conjecture in 2014 that, for all $k \geq 2$, its $k$-binomial complexity is the same as the usual factor complexity $2 n+1$ [18]. As in the Sturmian case, it is enough to show that given any two distinct factors of length $n$ occurring 
in the Tribonacci word, these two factors are not 2-binomially equivalent. Surprisingly, classical combinatorial techniques seemed to be unsuccessful. We make an extensive use of the concepts of templates and their ancestors similar to what can be found in [1, 2, 8] where avoidance of abelian repetitions is considered. Closely related, let us also mention [5] where a morphic word avoiding three consecutive factors of the same size and same sum is given. Recently F. Liétard proposed algorithmic proofs for morphic words avoiding additive powers [13].

This paper is organized as follows. In Section 2, we recall the notion of Parikh matrix and extend it to our $k$-binomial context. In particular, this extended matrix is built from the classical one using the Kronecker product: binomial coefficients can be nicely represented in terms of this product.

In Section 3 we define and adapt the notions of templates and ancestors to our purpose. To solve our problem, we need to show the finiteness of some set of realizable ancestors. To that end, in Section 4, we first get several bounds related to Parikh vectors of factors of the Tribonacci word. Consequently, we deduce bounds on the realizable ancestors. We put together the results of these last two sections to establish the main theorem in Section 5. Similarly to [1, 2, 8, 13, 15], our proof is a computer-assisted one.

\section{BASICS}

Let $\Sigma=\{0, \ldots, k-1\}$ be an ordered alphabet of size $k$. As mentionned in the introduction, it is enough to consider 2-binomial equivalence but everything in this section generalizes well to $k$-binomial equivalence.

Definition 5. Let $w$ be a finite word over $\Sigma$. We will make an extensive use of its extended Parikh vector denoted by $\Psi(w)$ and defined as follows. We set

$$
\Psi(w):=\left(|w|_{0}, \ldots,|w|_{k-1},\left(\begin{array}{c}
w \\
00
\end{array}\right),\left(\begin{array}{c}
w \\
01
\end{array}\right), \ldots,\left(\begin{array}{c}
w \\
(k-1)(k-1)
\end{array}\right)\right)^{\top} .
$$

It is a column vector of size $k(k+1)$ and we assume that the $k^{2}$ subwords of length 2 are lexicographically ordered.

Take the word $u=10010201010$ which is a factor of length 11 occurring in the Tribonacci word. Its extended Parikh vector is given by

$$
\Psi(u)=(6,4,1,15,11,3,13,6,2,3,2,0)^{\top} .
$$

Clearly, some of these entries are redundant, e.g., for all $a \in \Sigma$, ( $\left.\begin{array}{c}w \\ a a\end{array}\right)=$ $\left(\begin{array}{c}|w| a \\ 2\end{array}\right)$ where on the r.h.s. we have a classical binomial coefficient. With this notation, $\Psi(u)=\Psi(v)$ if and only if $u \sim_{2} v$.

For a vector $\mathbf{d} \in \mathbb{Z}^{n}, n \geq k$, we let $\left.\mathbf{d}\right|_{0, \ldots, k-1}$ denote the vector in $\mathbb{Z}^{k}$ made of the first $k$ coordinates of $\mathbf{d}$. In particular over an alphabet of size $k,\left.\Psi(w)\right|_{0, \ldots, k-1}=\Psi^{\prime}(w)$. 
We let $A \otimes B$ denote the usual Kronecker product of two matrices $A \in$ $\mathbb{Z}^{m \times n}$ and $B \in \mathbb{Z}^{p \times q}$. It is a block-matrix in $\mathbb{Z}^{m p \times n q}$ defined by

$$
A \otimes B:=\left(\begin{array}{ccc}
a_{11} B & \cdots & a_{1 n} B \\
\vdots & \ddots & \vdots \\
a_{m 1} B & \cdots & a_{m n} B
\end{array}\right) .
$$

Let us recall some well-known properties of the Kronecker product, see [9].

Lemma 6. For all matrices $A, B, C, D$, and for every scalar $k$, as long as the involved products and sums of matrices are defined, the following equalities hold:

- $(A \otimes B)(C \otimes D)=(A C) \otimes(B D)$,

- $(A+B) \otimes D=(A \otimes D)+(B \otimes D)$,

- $A \otimes(C+D)=(A \otimes C)+(A \otimes D)$,

- $k(A \otimes B)=(k A \otimes B)=(A \otimes k B)$,

- $\operatorname{det}(A \otimes B)=\operatorname{det}(A)^{m} \operatorname{det}(B)^{n}$ when $A$ and $B$ are square matrices of size $n$ and $m$ respectively.

Let $k$ be an integer. We let $P_{k} \in \mathbb{Z}^{(k(k+1)) \times k^{2}}$ denote the matrix such that for all, $i, j$,

$$
\left[P_{k}\right]_{i, j}= \begin{cases}1, & \text { if } i=j+k \\ 0, & \text { otherwise }\end{cases}
$$

This matrix adds $k$ zeros at the beginning of a column vector of size $k^{2}$. We start with two straightforward lemmas and the introduction of an extended Parikh matrix.

Lemma 7. Let $u$ and $v$ be two words over an alphabet of size $k$. We have

$$
\Psi(u v)=\Psi(u)+\Psi(v)+P_{k}\left(\Psi^{\prime}(u) \otimes \Psi^{\prime}(v)\right) .
$$

Proof. The first two terms in the statement take into account the separate contributions of $u$ and $v$ to the different coefficients. Nevertheless, subwords of length 2 can also be obtained by taking their first letter in $u$ and their second one in $v$. This is exactly the contribution of the third term. Observe that $\Psi^{\prime}(u) \otimes \Psi^{\prime}(v)$ is a column vector of size $k^{2}$. Applying $P_{k}$ will add $k$ zeros on top because the contribution of individual letters has already been taken into account in the first two terms.

The classical Parikh matrix $M_{h}^{\prime}$ associated with a morphism $h$ is a useful tool in combinatorics on words. Over an ordered $k$-letter alphabet, it is defined from its columns as a $k \times k$ matrix

$$
M_{h}^{\prime}=\left(\begin{array}{lll}
\Psi^{\prime}(h(0)) & \cdots & \Psi^{\prime}(h(k-1))
\end{array}\right)
$$

and it readily satisfies

$$
\Psi^{\prime}(h(u))=M_{h}^{\prime} \Psi^{\prime}(u), \quad \forall u \in \Sigma^{*} .
$$


For the Tribonacci morphism, it is given by

$$
M_{\tau}^{\prime}=\left(\begin{array}{lll}
1 & 1 & 1 \\
1 & 0 & 0 \\
0 & 1 & 0
\end{array}\right)
$$

Definition 8. Mimicking the Parikh matrix and its use, one can define an extended Parikh matrix $M_{h}$ associated with a morphism $h$ defined over an ordered $k$-letter alphabet. It is a $k(k+1) \times k(k+1)$ matrix satisfying

$$
\Psi(h(u))=M_{h} \Psi(u), \quad \forall u \in \Sigma^{*} .
$$

The existence of the extended Parikh matrix satisfying (2) is ensured by the next result.

Lemma 9. Let $M_{h}^{\prime}$ be the Parikh matrix associated with some morphism $h$. The extended Parikh matrix of $h$ has the following form:

$$
M_{h}=\left(\begin{array}{cccc}
M_{h}^{\prime} & 0 & \cdots & 0 \\
\star & & & \\
\star & & M_{h}^{\prime} \otimes M_{h}^{\prime} & \\
\star & & &
\end{array}\right) .
$$

In particular, $\operatorname{det}\left(M_{h}\right)=\operatorname{det}\left(M_{h}^{\prime}\right)^{2 k+1}$. Moreover, if the alphabet is of size $k$, then $M_{h} P_{k}=P_{k}\left(M_{h}^{\prime} \otimes M_{h}^{\prime}\right)$. If $M_{h}^{\prime}$ is non-singular, then $M_{h}$ is non-singular and $M_{h}^{-1}$ is a block-triangular matrix of the same form as $M_{h}$ with diagonal blocks $M_{h}^{\prime-1}$ and $M_{h}^{\prime-1} \otimes M_{h}^{\prime-1}$.

Proof. The first $k$ components of $\Psi(u)$ give the usual Parikh vector, hence $M_{h}^{\prime}$ is the upper-left corner of $M_{h}$. For the last $k^{2}$ components of $\Psi(u)$ dealing with binomial coefficients of subwords of length 2, it is shown in [12] that, for all $a, b \in \Sigma$,

$$
\left(\begin{array}{c}
h(u) \\
a b
\end{array}\right)=\sum_{c \in \Sigma}\left(\begin{array}{c}
h(c) \\
a b
\end{array}\right)|u|_{c}+\sum_{x_{1} x_{2} \in \Sigma^{2}}\left(\begin{array}{c}
h\left(x_{1}\right) \\
a
\end{array}\right)\left(\begin{array}{c}
h\left(x_{2}\right) \\
b
\end{array}\right)\left(\begin{array}{c}
u \\
x_{1} x_{2}
\end{array}\right) .
$$

In this expression, the first sum corresponds to the $3 \times 3$ submatrices marked as $\star$ and the second sum exactly corresponds to the Kronecker product $M_{h}^{\prime} \otimes$ $M_{h}^{\prime}$. Indeed, if we index $M_{h}^{\prime}$ on $\Sigma$ and $M_{h}^{\prime} \otimes M_{h}^{\prime}$ on $\Sigma^{2}$, we have

$$
\left(\begin{array}{c}
h\left(x_{1}\right) \\
a
\end{array}\right)\left(\begin{array}{c}
h\left(x_{2}\right) \\
b
\end{array}\right)=\left[M_{h}^{\prime}\right]_{a, x_{1}}\left[M_{h}^{\prime}\right]_{b, x_{2}}=\left[M_{h}^{\prime} \otimes M_{h}^{\prime}\right]_{a b, x_{1} x_{2}} .
$$

This extended Parikh matrix was also used in 14 (for avoidance problems).

We will use several times this lemma.

Lemma 10. Let $n, m \in \mathbb{N}^{+}, a \in \mathbb{C}, c, v_{1}, \ldots, v_{m} \in \mathbb{C}^{n}$ and $\mathcal{C}$ be the convex hull of $\left\{v_{1}, \ldots, v_{m}\right\}$. Then

$$
\max _{x \in \mathcal{C}}|a+c \cdot x|=\max _{x \in\left\{v_{1}, \ldots, v_{m}\right\}}|a+c \cdot x| .
$$


Proof. Let $x \in \mathcal{C}$ such that $|a+c \cdot x|=\max _{y \in \mathcal{C}}|a+c \cdot y|$. By definition, for any $x \in \mathcal{C}$, there are $\alpha_{1}, \ldots, \alpha_{m} \in[0,1]$ such that $1=\sum_{i \in\{1, \ldots, m\}} \alpha_{i}$ and $x=\sum_{i \in\{1, \ldots, m\}} \alpha_{i} v_{i}$. Then, using linearity and triangular inequality, we get:

$$
\begin{aligned}
\max _{y \in \mathcal{C}}|a+c \cdot y| & =|a+c \cdot x| \\
& =\left|a+c \cdot \sum_{i \in\{1, \ldots, m\}} \alpha_{i} v_{i}\right| \\
& =\left|\sum_{i \in\{1, \ldots, m\}}\left(\alpha_{i}\left(a+c \cdot v_{i}\right)\right)\right| \\
& \leq \sum_{i \in\{1, \ldots, m\}} \alpha_{i}\left|a+c \cdot v_{i}\right| \\
& \leq \sum_{i \in\{1, \ldots, m\}} \alpha_{i} \max _{y \in\left\{v_{1}, \ldots, v_{m}\right\}}|a+c \cdot y| \\
\max _{y \in \mathcal{C}}|a+c \cdot y| & \leq \max _{y \in\left\{v_{1}, \ldots, v_{m}\right\}}|a+c \cdot y| .
\end{aligned}
$$

Since $\left\{v_{1}, \ldots, v_{m}\right\} \subseteq \mathcal{C}, \max _{y \in \mathcal{C}}|a+c \cdot y|=\max _{y \in\left\{v_{1}, \ldots, v_{m}\right\}}|a+c \cdot y|$ which concludes the proof.

\section{Templates And AnCESTORS}

For this section, let $h: \Sigma^{*} \rightarrow \Sigma^{*}$ be any primitive (prolongeable) morphism. Let $M_{h}^{\prime}$ be its Parikh matrix and $M_{h}$ be its extended Parikh matrix. We let $k:=\# \Sigma$. Recall that a prefix (resp. a suffix) of a word $w$ is proper, if it is different from $w$ (and thus, possibly empty).

Definition 11. The language of $h$, denoted by $\mathcal{L}(h)$, is the set of factors of any of its non-empty fixed points (if $h$ is primitive, they all have the same language). The set $\operatorname{Pref}(h)$ (resp. Suff $(h))$ is the set of proper prefixes (resp. proper suffixes) of the words in $\{h(a) \mid a \in \Sigma\}$, e.g., for the Tribonacci morphism, $\operatorname{Pref}(\tau)=\{\varepsilon, 0\}$ and $\operatorname{Suff}(\tau)=\{\varepsilon, 1,2\}$. Such a notation can be extended to $h^{n}$. If $u \in \mathcal{L}(h)$, there exist a shortest $p_{u} \in \operatorname{Pref}(h)$, a shortest $s_{u} \in \operatorname{Suff}(h)$ and $u^{\prime} \in \mathcal{L}(h)$ such that $h\left(u^{\prime}\right)=p_{u} u s_{u}$.

In the following definition, the index $\mathbf{b}$ (resp., e) stands for beginning (resp., end).

Definition 12. A template is a 5-tuple of the form $t=\left[\mathbf{d}, \mathbf{D}_{\mathbf{b}}, \mathbf{D}_{\mathbf{e}}, a_{1}, a_{2}\right]$ where $a_{1}, a_{2} \in \Sigma, \mathbf{d} \in \mathbb{Z}^{k(k+1)}$ and $\mathbf{D}_{\mathbf{b}}, \mathbf{D}_{\mathbf{e}} \in \mathbb{Z}^{k}$. A pair of words $(u, v)$ is a realization of (or realizes) the template $t$ if:

- $\Psi(u)-\Psi(v)=\mathbf{d}+P_{k}\left(\mathbf{D}_{\mathbf{b}} \otimes \Psi^{\prime}(u)+\Psi^{\prime}(u) \otimes \mathbf{D}_{\mathbf{e}}\right)$,

- there exist $u^{\prime}$ and $v^{\prime}$ such that $u=u^{\prime} a_{1}$ and $v=v^{\prime} a_{2}$.

A template $t$ is realizable by $h$ if there is a pair of words in $\mathcal{L}(h)$ that realize $t$. 
Given two factors $u$ and $v$, the template of the form $\left[\Psi(u)-\Psi(v), \mathbf{0}, \mathbf{0}, a_{1}, a_{2}\right]$ is obviously realizable by $h$, where $a_{1}$ (resp. $a_{2}$ ) is the last letter of $u$ (resp. $v)$.

Due to the presence of $P_{k}$ in the above definition, note that if a template is realizable by a pair $(u, v)$, then the corresponding vector $\mathbf{d}$ is such that $\left.\mathbf{d}\right|_{0, \ldots, k-1}=\Psi^{\prime}(u)-\Psi^{\prime}(v)$.

Example. Take $u=00102010$ and $v=10201020$ two factors of the Tribonacci word. We have

$$
\begin{gathered}
\Psi(u)=(5,2,1,10,6,3,4,1,1,2,1,0)^{\top} \\
\Psi(v)=(4,2,2,6,2,4,6,1,3,4,1,1)^{\top}
\end{gathered}
$$

and the difference is given by

$$
\Psi(u)-\Psi(v)=(1,0,-1,4,4,-1,-2,0,-2,-2,0,-1)^{\top} .
$$

Taking

$$
\mathbf{d}=(1,0,-1,-1,-1,4,1,0,1,-3,-1,0)^{\top}
$$

and

leads to

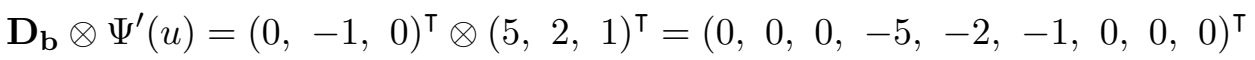

and

$\Psi^{\prime}(u) \otimes \mathbf{D}_{\mathbf{e}}=(5,2,1)^{\top} \otimes(1,1,-1)^{\top}=(5,5,-5,2,2,-2,1,1,-1)^{\top}$.

This shows that we have a template $\left[\mathbf{d}, \mathbf{D}_{\mathbf{b}}, \mathbf{D}_{\mathbf{e}}, 0,0\right]$ realizable by $\tau$. Actually, for any choice of words $u, v$ and vectors $\mathbf{D}_{\mathbf{b}}, \mathbf{D}_{\mathbf{e}}$ in $\mathbb{Z}^{k}$, there exists a convenient $\mathbf{d} \in \mathbb{Z}^{k(k+1)}$.

Lemma 13. Let $h$ be a primitive morphism. Let $T:=\left\{\left[\mathbf{0}, \mathbf{0}, \mathbf{0}, a_{1}, a_{2}\right]: a_{1} \neq\right.$ $\left.a_{2}\right\}$. The factorial complexity and the 2-binomial complexity of any fixed point of $h$ are equal if and only if all templates from $T$ are non-realizable by $h$.

Proof. The factorial complexity is not the same as the 2-binomial complexity if and only if there exists a pair of factors $(u, v)$ such that $u \neq v$ and $\Psi(u)=$ $\Psi(v)$.

The two words of any realization of an element in $T$ are 2-binomially equivalent and are different since they do not have the same last letter. Thus, if there is a realization of an element of $T$ then the factorial complexity and the 2-binomial complexity are not equal.

Now, for the other direction, suppose that the two complexity functions are not equal: we have a pair of words $(u, v)$ such that $u \neq v$ and $\Psi(u)=\Psi(v)$. Since $u \neq v$ and $|u|=|v|$, there exist $u^{\prime}, v^{\prime}, s \in \Sigma^{*}$ and $a, b \in \Sigma$ with $a \neq b$ such that $u=u^{\prime} a s$ and $v=v^{\prime} b s$ (observe that $s$ is the longest common suffix of $u$ and $v)$. Then $\Psi\left(u^{\prime} a\right)=\Psi\left(v^{\prime} b\right)$ so the pair $\left(u^{\prime} a, v^{\prime} b\right)$ realizes $[\mathbf{0}, \mathbf{0}, \mathbf{0}, a, b]$, which belongs to $T$. 
The next definition could at first glance seem artificial. Nevertheless, the reader will grasp its meaning after going through the proof of Lemma 15.

Definition 14. Let $t^{\prime}=\left[\mathbf{d}^{\prime}, \mathbf{D}_{\mathbf{b}}^{\prime}, \mathbf{D}_{\mathbf{e}}^{\prime}, a_{1}^{\prime}, a_{2}^{\prime}\right]$ and $t=\left[\mathbf{d}, \mathbf{D}_{\mathbf{b}}, \mathbf{D}_{\mathbf{e}}, a_{1}, a_{2}\right]$ be two templates and $h$ be a morphism. We say that $t^{\prime}$ is a parent by $h$ of $t$ if there exist $p_{u}, p_{v} \in \operatorname{Pref}(h)$ and $s_{u}, s_{v} \in \operatorname{Suff}(h)$ such that:

- $\mathbf{d}^{\prime}$ is given by

$$
\begin{aligned}
& M_{h} \mathbf{d}^{\prime}=\mathbf{d}+\Psi\left(p_{u} s_{u}\right)-\Psi\left(p_{v} s_{v}\right)+P_{k}\left(\left.\Psi^{\prime}\left(p_{v}\right) \otimes \mathbf{d}\right|_{0, \ldots, k-1}+\left.\mathbf{d}\right|_{0, \ldots, k-1} \otimes \Psi^{\prime}\left(s_{v}\right)\right) \\
- & P_{k}\left(\left(\mathbf{D}_{\mathbf{b}}+\Psi^{\prime}\left(p_{u}\right)-\Psi^{\prime}\left(p_{v}\right)\right) \otimes \Psi^{\prime}\left(p_{u} s_{u}\right)+\Psi^{\prime}\left(p_{u} s_{u}\right) \otimes\left(\mathbf{D}_{\mathbf{e}}+\Psi^{\prime}\left(s_{u}\right)-\Psi^{\prime}\left(s_{v}\right)\right) ;\right.
\end{aligned}
$$

- the value of $\mathbf{D}_{\mathbf{b}}^{\prime}$ is given by $M_{h}^{\prime} \mathbf{D}_{\mathbf{b}}^{\prime}=\mathbf{D}_{\mathbf{b}}+\Psi^{\prime}\left(p_{u}\right)-\Psi^{\prime}\left(p_{v}\right)$;

- the value of $\mathbf{D}_{\mathbf{e}}^{\prime}$ is given by $M_{h}^{\prime} \mathbf{D}_{\mathbf{e}}^{\prime}=\mathbf{D}_{\mathbf{e}}+\Psi^{\prime}\left(s_{u}\right)-\Psi^{\prime}\left(s_{v}\right)$;

- $a_{1} s_{u}$ is a suffix of $h\left(a_{1}^{\prime}\right)$;

- $a_{2} s_{u}$ is a suffix of $h\left(a_{2}^{\prime}\right)$.

We let $\operatorname{Par}_{h}(t)$ denote the set of parents by $h$ of $t$.

Remark. Observe that for any given template $t, \operatorname{Par}_{h}(t)$ is finite and easy to compute as long as $M_{h}$ and $M_{h}^{\prime}$ are non-singular. Indeed, the sets $\operatorname{Pref}(h)$ and $\operatorname{Suff}(h)$ are finite. For the Tribonacci word, $\# \operatorname{Pref}(\tau)=2$, \# $\operatorname{Suff}(\tau)=$ 3 , hence $\# \operatorname{Par}_{\tau}(t) \leq 36$. At this stage, it is not required for a parent to be realizable.

More interestingly there is a link between preimages of the realization by $h$ of a template and realization by $h$ of the parents of the template. We make that link explicit in the following Lemma.

Lemma 15. Let $h$ be a morphism. Assume that $\operatorname{det}\left(M_{h}^{\prime}\right)= \pm 1$. Let $t$ be a template, $u, v, v^{\prime}, u^{\prime} \in \mathcal{L}(h), p_{u}, p_{v} \in \operatorname{Pref}(h)$ and $s_{u}, s_{v} \in \operatorname{Suff}(h)$ such that:

- $h\left(u^{\prime}\right)=p_{u} u s_{u}$ and $h\left(v^{\prime}\right)=p_{v} v s_{v}$;

- $s_{u}$ is a proper suffix of the image of the last letter of $u^{\prime}$;

- $s_{v}$ is a proper suffix of the image of the last letter of $v^{\prime}$;

- $(u, v)$ realizes $t$.

Then there exists a parent $t^{\prime}$ of $t$ such that $\left(u^{\prime}, v^{\prime}\right)$ realizes $t^{\prime}$.

Proof. Let $t=\left[\mathbf{d}, \mathbf{D}_{\mathbf{b}}, \mathbf{D}_{\mathbf{e}}, a_{1}, a_{2}\right]$ be a template realized by $(u, v)$. Let us compute $M_{h}\left(\Psi\left(u^{\prime}\right)-\Psi\left(v^{\prime}\right)\right)$. We first make use of (2):

$$
\begin{aligned}
M_{h}\left(\Psi\left(u^{\prime}\right)-\Psi\left(v^{\prime}\right)\right)= & \Psi\left(h\left(u^{\prime}\right)\right)-\Psi\left(h\left(v^{\prime}\right)\right)=\Psi\left(p_{u} u s_{u}\right)-\Psi\left(p_{v} v s_{v}\right) \\
= & \Psi(u)-\Psi(v)+\Psi\left(p_{u} s_{u}\right)-\Psi\left(p_{v} s_{v}\right) \\
& +P_{k}\left(\Psi^{\prime}\left(p_{u}\right) \otimes \Psi^{\prime}(u)+\Psi^{\prime}(u) \otimes \Psi^{\prime}\left(s_{u}\right)\right) \\
& -P_{k}\left(\Psi^{\prime}\left(p_{v}\right) \otimes \Psi^{\prime}(v)+\Psi^{\prime}(v) \otimes \Psi^{\prime}\left(s_{v}\right)\right) .
\end{aligned}
$$

The last line comes from the fact that, for all $a, b \in \Sigma$,

$$
\left(\begin{array}{c}
p_{u} u s_{u} \\
a b
\end{array}\right)=\left(\begin{array}{c}
p_{u} s_{u} \\
a b
\end{array}\right)+\left(\begin{array}{c}
u \\
a b
\end{array}\right)+\left|p_{u}\right|_{a}|u|_{b}+|u|_{a}\left|s_{u}\right|_{b} .
$$


Now we can use the equality $\Psi^{\prime}(v)=\Psi^{\prime}(u)-\left.\mathbf{d}\right|_{0, \ldots, k-1}$ and the definition of a template to express $\Psi(u)-\Psi(v)$ from $t$. The vector $M_{h}\left(\Psi\left(u^{\prime}\right)-\Psi\left(v^{\prime}\right)\right)$ is equal to

$$
\begin{aligned}
& \mathbf{d}+P_{k}\left(\mathbf{D}_{\mathbf{b}} \otimes \Psi^{\prime}(u)+\Psi^{\prime}(u) \otimes \mathbf{D}_{\mathbf{e}}\right)+\Psi\left(p_{u} s_{u}\right)-\Psi\left(p_{v} s_{v}\right) \\
& +P_{k}\left(\Psi^{\prime}\left(p_{u}\right) \otimes \Psi^{\prime}(u)+\Psi^{\prime}(u) \otimes \Psi^{\prime}\left(s_{u}\right)\right) \\
& -P_{k}\left(\Psi^{\prime}\left(p_{v}\right) \otimes \Psi^{\prime}(u)+\Psi^{\prime}(u) \otimes \Psi^{\prime}\left(s_{v}\right)-\left.\Psi^{\prime}\left(p_{v}\right) \otimes \mathbf{d}\right|_{0, \ldots, k-1}-\left.\mathbf{d}\right|_{0, \ldots, k-1} \otimes \Psi^{\prime}\left(s_{v}\right)\right) .
\end{aligned}
$$

Rearranging the terms gives us a new expression of $M_{h}\left(\Psi\left(u^{\prime}\right)-\Psi\left(v^{\prime}\right)\right)$ :

$$
\begin{aligned}
& \mathbf{d}+\Psi\left(p_{u} s_{u}\right)-\Psi\left(p_{v} s_{v}\right)+P_{k}\left(\left.\Psi^{\prime}\left(p_{v}\right) \otimes \mathbf{d}\right|_{0, \ldots, k-1}+\left.\mathbf{d}\right|_{0, \ldots, k-1} \otimes \Psi^{\prime}\left(s_{v}\right)\right) \\
& +P_{k}\left(\left(\mathbf{D}_{\mathbf{b}}+\Psi^{\prime}\left(p_{u}\right)-\Psi^{\prime}\left(p_{v}\right)\right) \otimes \Psi^{\prime}(u)+\Psi^{\prime}(u) \otimes\left(\mathbf{D}_{\mathbf{e}}+\Psi^{\prime}\left(s_{u}\right)-\Psi^{\prime}\left(s_{v}\right)\right)\right. \\
= & \mathbf{d}+\Psi\left(p_{u} s_{u}\right)-\Psi\left(p_{v} s_{v}\right)+P_{k}\left(\left.\Psi^{\prime}\left(p_{v}\right) \otimes \mathbf{d}\right|_{0, \ldots, k-1}+\left.\mathbf{d}\right|_{0, \ldots, k-1} \otimes \Psi^{\prime}\left(s_{v}\right)\right) \\
& -P_{k}\left(\left(\mathbf{D}_{\mathbf{b}}+\Psi^{\prime}\left(p_{u}\right)-\Psi^{\prime}\left(p_{v}\right)\right) \otimes \Psi^{\prime}\left(p_{u} s_{u}\right)+\Psi^{\prime}\left(p_{u} s_{u}\right) \otimes\left(\mathbf{D}_{\mathbf{e}}+\Psi^{\prime}\left(s_{u}\right)-\Psi^{\prime}\left(s_{v}\right)\right)\right. \\
& +P_{k}\left(\left(\mathbf{D}_{\mathbf{b}}+\Psi^{\prime}\left(p_{u}\right)-\Psi^{\prime}\left(p_{v}\right)\right) \otimes \Psi^{\prime}\left(p_{u} u s_{u}\right)+\Psi^{\prime}\left(p_{u} u s_{u}\right) \otimes\left(\mathbf{D}_{\mathbf{e}}+\Psi^{\prime}\left(s_{u}\right)-\Psi^{\prime}\left(s_{v}\right)\right)\right.
\end{aligned}
$$

where for the last line, we simply recall that $\Psi^{\prime}(u)=\Psi^{\prime}\left(p_{u} u s_{u}\right)-\Psi^{\prime}\left(p_{u} s_{u}\right)$. Since $\operatorname{det}\left(M_{h}^{\prime}\right)= \pm 1$, we also have $\operatorname{det}\left(M_{h}\right)= \pm 1$ and thus the following vector has integer entries

$$
\begin{gathered}
\quad \mathbf{d}^{\prime}:=M_{h}^{-1}\left(\mathbf{d}+\Psi\left(p_{u} s_{u}\right)-\Psi\left(p_{v} s_{v}\right)+P_{k}\left(\left.\Psi^{\prime}\left(p_{v}\right) \otimes \mathbf{d}\right|_{0, \ldots, k-1}+\left.\mathbf{d}\right|_{0, \ldots, k-1} \otimes \Psi^{\prime}\left(s_{v}\right)\right)\right. \\
-P_{k}\left(\left(\mathbf{D}_{\mathbf{b}}+\Psi^{\prime}\left(p_{u}\right)-\Psi^{\prime}\left(p_{v}\right)\right) \otimes \Psi^{\prime}\left(p_{u} s_{u}\right)+\Psi^{\prime}\left(p_{u} s_{u}\right) \otimes\left(\mathbf{D}_{\mathbf{e}}+\Psi^{\prime}\left(s_{u}\right)-\Psi^{\prime}\left(s_{v}\right)\right)\right) .
\end{gathered}
$$

Then, recalling the form of $M_{h}$ given in Lemma 9 and classical properties of Kronecker product (Lemma 6), we get

$$
\begin{aligned}
\Psi\left(u^{\prime}\right)-\Psi\left(v^{\prime}\right)= & \mathbf{d}^{\prime}+M_{h}^{-1} P_{k}\left(\left(\mathbf{D}_{\mathbf{b}}+\Psi^{\prime}\left(p_{u}\right)-\Psi^{\prime}\left(p_{v}\right)\right) \otimes \Psi^{\prime}\left(p_{u} u s_{u}\right)\right. \\
& \left.+\Psi^{\prime}\left(p_{u} u s_{u}\right) \otimes\left(\mathbf{D}_{\mathbf{e}}+\Psi^{\prime}\left(s_{u}\right)-\Psi^{\prime}\left(s_{v}\right)\right)\right) \\
= & \mathbf{d}^{\prime}+P_{k}\left(\left(M_{h}^{\prime-1} \otimes M_{h}^{\prime-1}\right)\left(\left(\mathbf{D}_{\mathbf{b}}+\Psi^{\prime}\left(p_{u}\right)-\Psi^{\prime}\left(p_{v}\right)\right) \otimes \Psi^{\prime}\left(p_{u} u s_{u}\right)\right)\right. \\
& +\left(M_{h}^{\prime-1} \otimes M_{h}^{\prime-1}\right)\left(\Psi^{\prime}\left(p_{u} u s_{u}\right) \otimes\left(\mathbf{D}_{\mathbf{e}}+\Psi^{\prime}\left(s_{u}\right)-\Psi^{\prime}\left(s_{v}\right)\right)\right) \\
= & \mathbf{d}^{\prime}+P_{k}\left(\left(M_{h}^{\prime-1}\left(\mathbf{D}_{\mathbf{b}}+\Psi^{\prime}\left(p_{u}\right)-\Psi^{\prime}\left(p_{v}\right)\right)\right) \otimes\left(M_{h}^{\prime-1} \Psi^{\prime}\left(p_{u} u s_{u}\right)\right)\right. \\
& +\left(M_{h}^{\prime-1} \Psi^{\prime}\left(p_{u} u s_{u}\right)\right) \otimes\left(M_{h}^{\prime-1}\left(\mathbf{D}_{\mathbf{e}}+\Psi^{\prime}\left(s_{u}\right)-\Psi^{\prime}\left(s_{v}\right)\right)\right) \\
= & \mathbf{d}^{\prime}+P_{k}\left(\left(M_{h}^{\prime-1}\left(\mathbf{D}_{\mathbf{b}}+\Psi^{\prime}\left(p_{u}\right)-\Psi^{\prime}\left(p_{v}\right)\right)\right) \otimes \Psi^{\prime}\left(u^{\prime}\right)\right. \\
& \left.+\Psi^{\prime}\left(u^{\prime}\right) \otimes\left(M_{h}^{\prime-1}\left(\mathbf{D}_{\mathbf{e}}+\Psi^{\prime}\left(s_{u}\right)-\Psi^{\prime}\left(s_{v}\right)\right)\right)\right)
\end{aligned}
$$

where on the last line, we make use of (2). Finally, if we let

$$
\mathbf{D}_{\mathbf{b}}^{\prime}:=M_{h}^{\prime-1}\left(\mathbf{D}_{\mathbf{b}}+\Psi^{\prime}\left(p_{u}\right)-\Psi^{\prime}\left(p_{v}\right)\right)
$$

and

we get

$$
\mathbf{D}_{\mathbf{e}}^{\prime}:=M_{h}^{\prime-1}\left(\mathbf{D}_{\mathbf{e}}+\Psi^{\prime}\left(s_{u}\right)-\Psi^{\prime}\left(s_{v}\right)\right)
$$

$$
\Psi\left(u^{\prime}\right)-\Psi\left(v^{\prime}\right)=\mathbf{d}^{\prime}+P_{k}\left(\mathbf{D}_{\mathbf{b}}^{\prime} \otimes \Psi^{\prime}\left(u^{\prime}\right)+\Psi^{\prime}\left(u^{\prime}\right) \otimes \mathbf{D}_{\mathbf{e}}^{\prime}\right)
$$


Let $a_{1}^{\prime}$ be the last letter of $u^{\prime}$, and $a_{2}^{\prime}$ be the last letter of $v^{\prime}$. Since $s_{u}$ is a proper suffix of $h\left(a_{1}^{\prime}\right), a_{1} s_{u}$ is a suffix of $h\left(a_{1}^{\prime}\right)$. Similarly $a_{2} s_{u}$ is a suffix of $h\left(a_{2}^{\prime}\right)$.

From the definitions of realizable template and parent, it is clear that the template $t^{\prime}=\left[\mathbf{d}^{\prime}, \mathbf{D}_{\mathbf{b}}^{\prime}, \mathbf{D}_{\mathbf{e}}^{\prime}, a_{1}^{\prime}, a_{2}^{\prime}\right]$ is a parent of $t$ and is realized by $\left(u^{\prime}, v^{\prime}\right)$.

This motivates the following definitions.

Definition 16. A template $t^{\prime}$ is an ancestor by $h$ (resp., realizable ancestor) of a template $t$ if there exists a sequence of $n \geq 1$ templates (resp., realizable templates) $t=t_{1}, t_{2}, \ldots, t_{n}=t^{\prime}$ such that for all $i \in\{1, \ldots, n-1\}, t_{i+1}$ is a parent by $h$ of $t_{i}$. For a template $t$, we denote by $\mathrm{RAnc}_{h}(t)$ the set of all the realizable ancestors by $h$ of $t$. We may omit "by $h$ " when the morphism is clear from the context.

Example. Take the words $u=00102010$ and $v=10201020$ and the template of the previous example. Let $u^{\prime}=20100$ and $v^{\prime}=01010$ be two factors of the Tribonacci word. We have

$$
h\left(u^{\prime}\right)=u 1, h\left(v^{\prime}\right)=0 v 1
$$

and we set $p_{u}=\varepsilon, s_{u}=1, p_{v}=0$ and $s_{v}=1$. Using Definition 14, we compute

$$
\mathbf{D}_{\mathbf{b}}^{\prime}=(-1,0,0)^{\top} \quad \text { and } \mathbf{D}_{\mathbf{e}}^{\prime}=(1,-1,1)^{\top} \text {. }
$$

Hence, we get

$$
\mathbf{d}^{\prime}=(0,-1,1,0,-2,0,-1,-1,0,3,1,0)^{\top}
$$

and by Lemma 15, this parent template is a template realized by $\left(u^{\prime}, v^{\prime}\right)$. It is a realizable ancestor. Applying (3) leads to

$$
\Psi\left(u^{\prime}\right)-\Psi\left(v^{\prime}\right)=(0,-1,1,0,-2,0,-1,-1,0,3,1,0)^{\top} .
$$

Since 2010 is a right-special factor of the Tribonacci word, we could also have chosen $u^{\prime}=20101$ (or, 20102). In that case, this changes $s_{u}$ to 2 (or, $\varepsilon$ ) and thus leads to other vectors $\mathbf{D}_{\mathbf{b}}^{\prime}, \mathbf{D}_{\mathbf{e}}^{\prime}, \mathbf{d}^{\prime}$ and other parent templates realizable by $\tau$.

Let $|h|=\max _{a \in \Sigma}|h(a)|$, usually called the width of $h$.

Proposition 17. Let $L$ be a positive integer. Let $h$ be a primitive morphism and $t_{0}$ be a template. If there exists a pair of words in $\mathcal{L}(h)$ that is a realization of $t_{0}$, then

- either $_{0}$ has a realization $(u, v) \in \mathcal{L}(h) \times \mathcal{L}(h)$ such that $\min (|u|,|v|) \leq$ $L$ or,

- there exists a realization $(u, v) \in \mathcal{L}(h) \times \mathcal{L}(h)$ of a template $t$ of $\operatorname{RAnc}_{h}\left(t_{0}\right)$ with $L \leq \min (|u|,|v|) \leq|h| L$.

Proof. Let $(u, v)$ be a pair of factors of $\mathcal{L}(h)$ realizing $t_{0}$. If $\min (|u|,|v|) \leq L$, there is nothing left to prove. Assume therefore that $\min (|u|,|v|)>L$.

Since $v$ is a factor of $\mathcal{L}(h)$, there are sequences of words $v=v_{1}, v_{2}, \ldots, v_{n} \in \Sigma^{*}$, $p_{1}, \ldots, p_{n-1} \in \operatorname{Pref}(h)$ and $s_{1}, \ldots, s_{n-1} \in \operatorname{Suff}(h)$ such that, for all $i<n$, 
$h\left(v_{i+1}\right)=p_{i} v_{i} s_{i}$ and $L \leq\left|v_{n}\right| \leq|h| L$. Moreover we may force that, for all $i<n, s_{i}$ is a proper suffix of the last letter of $v_{i+1}$.

Similarly, since $u$ is a factor of $\mathcal{L}(h)$, there are sequences of words $u=u_{1}$, $u_{2}, \ldots, u_{\ell} \in \Sigma^{*}$ and $p_{1}^{\prime}, \ldots, p_{\ell-1}^{\prime} \in \operatorname{Pref}(h)$ and $s_{1}^{\prime}, \ldots, s_{\ell-1}^{\prime} \in \operatorname{Suff}(h)$ such that, for all $i<\ell, h\left(u_{i+1}\right)=p_{i}^{\prime} u_{i} s_{i}^{\prime}$ and $L \leq\left|u_{\ell}\right| \leq L|h|$.

Let $m=\min (n, \ell)$. We can simply apply Lemma 15 inductively $m$ times. We obtain a template $t^{\prime}$ which is an ancestor of $t_{0}$ and is realized by $\left(u_{m}, v_{m}\right)$. Since $m=\min (n, \ell), L \leq \min \left(\left|u_{m}\right|,\left|v_{m}\right|\right) \leq|h| L$. This concludes the proof.

\section{Bounding ReAlizable templates for the Tribonacci WORD}

Let $\tau$ be the Tribonacci morphism and $\mathcal{T}$ be the Tribonacci word. The matrix $M_{\tau}^{\prime}$ was given in (1). Since it is primitive, we may use Perron's theorem. Densities of letters 0,1,2 exist and are denoted respectively by $\alpha_{0}, \alpha_{1}$ and $\alpha_{2}$. Let $\theta \approx 1.839$ be the Perron eigenvalue of $\tau$. Recall that $\boldsymbol{\alpha}=\left(\begin{array}{lll}\alpha_{0} & \alpha_{1} & \alpha_{2}\end{array}\right)^{\top}$ is an eigenvector of $\tau$ associated with $\theta$.

4.1. Bounds on extended Parikh vectors. From [16, Prop. 2.4], we know that, for every factor $w$ of $\mathcal{T}$,

$$
\left.|| w\right|_{i}-\alpha_{i}|w| \mid<1.5, \quad i \in\{0,1,2\} .
$$

Let $\Delta=\left\{\left(\delta_{0}, \delta_{1}\right):-1.5 \leq \delta_{0}, \delta_{1}, \delta_{0}+\delta_{1} \leq 1.5\right\}$. We will use this result in the following form:

Corollary 18. For every factor $w$ of $\mathcal{T}$, there exists $\left(\delta_{0}, \delta_{1}\right) \in \Delta$ such that

$$
\Psi^{\prime}(w)=|w|\left(\begin{array}{l}
\alpha_{0} \\
\alpha_{1} \\
\alpha_{2}
\end{array}\right)+\left(\begin{array}{c}
\delta_{0} \\
\delta_{1} \\
-\delta_{0}-\delta_{1}
\end{array}\right) .
$$

We deduce the following lemma.

Lemma 19. For all factors $w$ of the Tribonacci word, we have

$$
|| \tau(w)|-\theta| w|| \leq 1.5 .
$$

Proof. From Corollary 18 , there exists $\left(\delta_{0}, \delta_{1}\right) \in \Delta$ such that

$$
\Psi^{\prime}(w)=|w|\left(\begin{array}{l}
\alpha_{0} \\
\alpha_{1} \\
\alpha_{2}
\end{array}\right)+\left(\begin{array}{c}
\delta_{0} \\
\delta_{1} \\
-\delta_{0}-\delta_{1}
\end{array}\right)
$$

We thus have

$$
\begin{aligned}
\Psi^{\prime}(\tau(w))=M_{\tau}^{\prime} \Psi^{\prime}(w) & =|w| M_{\tau}^{\prime}\left(\begin{array}{c}
\alpha_{0} \\
\alpha_{1} \\
\alpha_{2}
\end{array}\right)+M_{\tau}^{\prime}\left(\begin{array}{c}
\delta_{0} \\
\delta_{1} \\
-\delta_{0}-\delta_{1}
\end{array}\right) \\
& =|w| \theta\left(\begin{array}{c}
\alpha_{0} \\
\alpha_{1} \\
\alpha_{2}
\end{array}\right)+\left(\begin{array}{c}
0 \\
\delta_{0} \\
\delta_{1}
\end{array}\right) .
\end{aligned}
$$


The length of $\tau(w)$ is obtained by computing the dot product of this result with $\left(\begin{array}{lll}1 & 1 & 1\end{array}\right)$. Hence, we get

$$
|\tau(w)|=\left(\alpha_{0}+\alpha_{1}+\alpha_{2}\right) \theta|w|+\delta_{0}+\delta_{1}=\theta|w|+\delta_{0}+\delta_{1} .
$$

Corollary 20. Let $w$ be a factor of $\mathcal{T}$. For all non-negative integers $n$, we have

$$
|| \tau^{n}(w)\left|-\theta^{n}\right| w|| \leq \frac{1.5 \theta^{n}}{\theta-1} .
$$

Proof. Since $\theta>1$, the formula is clearly true for $n=0$. We proceed by induction on $n$. First, using of the triangular inequality gives

$$
\begin{aligned}
& || \tau^{n}(w)\left|-\theta^{n}\right| w||=\left|\left(\left|\tau^{n}(w)\right|-\theta^{n-1}|\tau(w)|\right)+\left(\theta^{n-1}|\tau(w)|-\theta^{n}|w|\right)\right| \\
& || \tau^{n}(w)\left|-\theta^{n}\right| w|| \leq|| \tau^{n-1}(\tau(w))\left|-\theta^{n-1}\right| \tau(w)||+\theta^{n-1}|| \tau(w)|-\theta| w|| .
\end{aligned}
$$

Using the induction hypothesis on the first term and Lemma 19 on the second term, we get

$$
|| \tau^{n}(w)\left|-\theta^{n}\right| w|| \leq \frac{1.5 \theta^{n-1}}{\theta-1}+\theta^{n-1} 1.5 \leq \frac{1.5 \theta^{n}}{\theta-1}
$$

As an immediate corollary, we make note of the following fact.

Corollary 21. Let $w$ be a factor of $\mathcal{T}$. For all non-negative integers $n$, we have

$$
|w| \geq \frac{\left|\tau^{n}(w)\right|}{\theta^{n}}-\frac{1.5}{\theta-1} .
$$

The following property will also be useful. Note that all along the paper, we will use left eigenvectors of $M_{\tau}$.

Lemma 22. Let $\lambda$ be an eigenvalue of $M_{\tau}$ such that $|\lambda|<1$ and let $\mathbf{r}$ be a left eigenvector of $M_{\tau}$ associated with $\lambda$. Then for every word $w \in\{0,1,2\}^{*}$,

$$
\mathbf{r} \cdot P_{3}\left(\Psi^{\prime}(w) \otimes\left(\begin{array}{lll}
\alpha_{0} & \alpha_{1} & \alpha_{2}
\end{array}\right)^{\top}\right)=\mathbf{r} \cdot P_{3}\left(\left(\begin{array}{lll}
\alpha_{0} & \alpha_{1} & \alpha_{2}
\end{array}\right)^{\top} \otimes \Psi^{\prime}(w)\right)=0 .
$$

Proof. By linearity of the Kronecker product, it is enough to show that this is true for every letter $a$. Recall that the matrix $M_{\tau}$ is given by Lemma 9 and equality (1). One can check that its characteristic polynomial can be factorized as

$$
\left(X^{3}-3 X^{2}-X-1\right)\left(X^{3}-X^{2}-X-1\right)\left(X^{3}+X^{2}+X-1\right)^{2} .
$$

The first factor has roots $\theta^{2} \approx 3.382$ and two complex conjugates of modulus less than 1 . The second factor is the characteristic polynomial of $M_{\tau}^{\prime}$. It has $\theta$ as dominant root and two complex conjugate roots of modulus less than 1 . Finally, the third polynomial has a real root $\approx .543$ and two complex conjugate roots of modulus larger than 1 . Thus we have four simple eigenvalues of modulus less than 1 and one eigenvalue of geometric multiplicity 2. One can find six linearly independent eigenvectors such that any left eigenvector $\mathbf{r}$ of $M_{\tau}$ is a linear combination of these vectors. All these (exact) computations can be carried on in a Computer Algebra System such as Mathematica: 


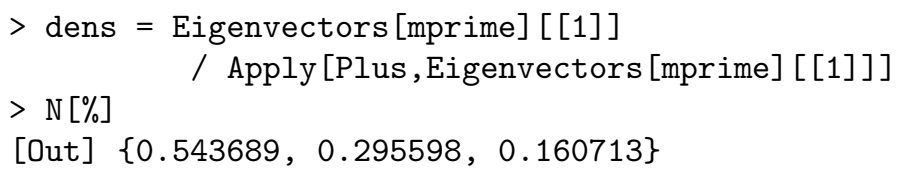

Let $j$ be the index of an eigenvalue of modulus less than one. We use the transpose of $M_{\tau}$ to take into account left eigenvectors.

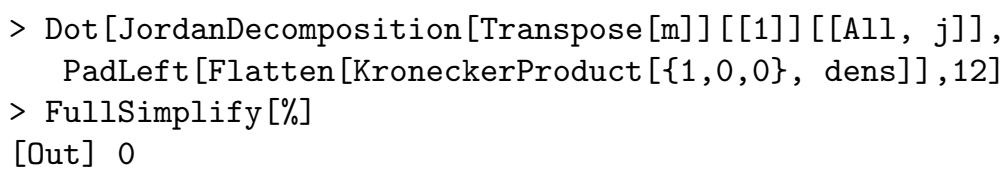

Remark, that this is not a lucky coincidence. It comes from the fact that $\left(\begin{array}{lll}\alpha_{0} & \alpha_{1} & \alpha_{2}\end{array}\right)$ is a right eigenvector of $M_{\tau}^{\prime}$ and that all the left eigenvectors of $M_{\tau}$ can be expressed from the left eigenvectors of $M_{\tau}^{\prime}$ using the Kronecker product.

We can show a first bound.

Lemma 23. Let $\mathbf{r}$ be a row vector in $\mathbb{C}^{12}$. For all integers $n$, words $p \in \operatorname{Pref}\left(\tau^{n}\right)$ and $s \in \operatorname{Suff}\left(\tau^{n}\right)$, there exist two positive real numbers $c_{1}(\mathbf{r}, p, s)$ and $c_{2}(\mathbf{r}, p, s)$ such that, for all words $w$, if pws is a factor of $\mathcal{T}$ then

$\left|\mathbf{r} \cdot\left(\Psi(p s)+P_{3}\left(\Psi^{\prime}(p) \otimes \Psi^{\prime}(w)+\Psi^{\prime}(w) \otimes \Psi^{\prime}(s)\right)\right)\right| \leq c_{1}(\mathbf{r}, p, s)|w|+c_{2}(\mathbf{r}, p, s)$.

Moreover, if

$$
\mathbf{r} \cdot P_{3}\left(\Psi^{\prime}(p) \otimes\left(\begin{array}{lll}
\alpha_{0} & \alpha_{1} & \alpha_{2}
\end{array}\right)^{\top}+\left(\begin{array}{lll}
\alpha_{0} & \alpha_{1} & \alpha_{2}
\end{array}\right)^{\top} \otimes \Psi^{\prime}(s)\right)=0,
$$

then $c_{1}(\mathbf{r}, p, s)=0$.

Proof. Let

$$
\left(\begin{array}{l}
\delta_{0} \\
\delta_{1} \\
\delta_{2}
\end{array}\right)=\Psi^{\prime}(w)-|w|\left(\begin{array}{l}
\alpha_{0} \\
\alpha_{1} \\
\alpha_{2}
\end{array}\right) .
$$

For all $p^{\prime} \in \operatorname{Suff}(p)$ and $s^{\prime} \in \operatorname{Pref}(s), p^{\prime} w s^{\prime}$ is a factor of $\mathcal{T}$. From Corollary 18 , we deduce that for all $i \in\{0,1,2\}$,

$$
\begin{aligned}
& -1.5 \leq\left|p^{\prime} w s^{\prime}\right|_{i}-\alpha_{i}\left|p^{\prime} w s^{\prime}\right| \leq 1.5 \\
& -1.5 \leq\left|p^{\prime} s^{\prime}\right|_{i}-\alpha_{i}\left|p^{\prime} s^{\prime}\right|+\delta_{i} \leq 1.5 \\
& -1.5-\left|p^{\prime} s^{\prime}\right|_{i}+\alpha_{i}\left|p^{\prime} s^{\prime}\right| \leq \delta_{i} \leq 1.5-\left|p^{\prime} s^{\prime}\right|_{i}+\alpha_{i}\left|p^{\prime} s^{\prime}\right| .
\end{aligned}
$$

Let

and

$$
l_{i}=\max _{\substack{s^{\prime} \in \operatorname{Pref}(s) \\ p^{\prime} \in \operatorname{Suff}(p)}}-1.5-\left|p^{\prime} s^{\prime}\right|_{i}+\alpha_{i}\left|p^{\prime} s^{\prime}\right|
$$

$$
u_{i}=\min _{\substack{s^{\prime} \in \operatorname{Pref}(s) \\ p^{\prime} \in \operatorname{Suff}(p)}} 1.5-\left|p^{\prime} s^{\prime}\right|_{i}+\alpha_{i}\left|p^{\prime} s^{\prime}\right| .
$$

Then, we can deduce the following bounds: $l_{i} \leq \delta_{i} \leq u_{i}$. 
For the sake of notation, we write $\boldsymbol{\alpha}=\left(\begin{array}{lll}\alpha_{0} & \alpha_{1} & \alpha_{2}\end{array}\right)^{\top}$ and $\boldsymbol{\delta}=\left(\begin{array}{lll}\delta_{0} & \delta_{1} & \delta_{2}\end{array}\right)^{\top}$. It gives

$$
\begin{aligned}
& \left|\mathbf{r} \cdot\left(\Psi(p s)+P_{3}\left(\Psi^{\prime}(p) \otimes \Psi^{\prime}(w)+\Psi^{\prime}(w) \otimes \Psi^{\prime}(s)\right)\right)\right| \\
= & \left|\mathbf{r} \cdot\left(\Psi(p s)+P_{3}\left(\Psi^{\prime}(p) \otimes[\boldsymbol{\alpha}|w|+\boldsymbol{\delta}]+[\boldsymbol{\alpha}|w|+\boldsymbol{\delta}] \otimes \Psi^{\prime}(s)\right)\right)\right| \\
\leq & \underbrace{\left|\mathbf{r} \cdot\left(\Psi(p s)+P_{3}\left(\Psi^{\prime}(p) \otimes \boldsymbol{\delta}+\boldsymbol{\delta} \otimes \Psi^{\prime}(s)\right)\right)\right|}_{=: f\left(\mathbf{r}, p, s, \delta_{0}, \delta_{1}, \delta_{2}\right)}+|w| \underbrace{\left|\mathbf{r} \cdot P_{3}\left(\Psi^{\prime}(p) \otimes \boldsymbol{\alpha}+\boldsymbol{\alpha} \otimes \Psi^{\prime}(s)\right)\right|}_{=: c_{1}(\mathbf{r}, p, s)} .
\end{aligned}
$$

We need $c_{2}(\mathbf{r}, p, s)$ to bound $f$ for all possible values of the $\delta_{i}$, so we can take

$$
c_{2}(\mathbf{r}, p, s):=\max _{\substack{l_{0} \leq \delta_{0} \leq u_{0} \\ l_{1} \leq \delta_{1} \leq u_{1} \\ l_{2} \leq \delta_{2} \leq u_{2} \\ \delta_{0}+\delta_{1}+\delta_{2}=0}} f\left(\mathbf{r}, p, s, \delta_{0}, \delta_{1}, \delta_{2}\right) .
$$

Then,

$\left|\mathbf{r} \cdot\left(\Psi(p s)+\Psi^{\prime}(p) \otimes \Psi^{\prime}(w)+\Psi^{\prime}(w) \otimes \Psi^{\prime}(s)\right)\right| \leq c_{1}(\mathbf{r}, p, s)|w|+c_{2}(\mathbf{r}, p, s)$.

Observe that

$$
\begin{aligned}
& \max _{\substack{l_{0} \leq \delta_{0} \leq u_{0} \\
l_{1} \leq \delta_{1} \leq u_{1} \\
l_{2} \leq \delta_{2} \leq u_{2} \\
\delta_{0}+\delta_{1}+\delta_{2}=0}} f\left(\mathbf{r}, p, s, \delta_{0}, \delta_{1}, \delta_{2}\right) \leq \\
& \min \left\{\max _{\substack{l_{0} \leq \delta_{0} \leq u_{0} \\
l_{1} \leq \delta_{1} \leq u_{1} \\
l_{2} \leq \delta_{2} \leq u_{2}}} f\left(\mathbf{r}, p, s, \delta_{0}, \delta_{1}, \delta_{2}\right), \max _{\substack{l_{0} \leq \delta_{0} \leq u_{0} \\
l_{1} \leq \delta_{1} \leq u_{1}}} f\left(\mathbf{r}, p, s, \delta_{0}, \delta_{1},-\delta_{0}-\delta_{1}\right),\right. \\
& \left.\max _{\substack{l_{0} \leq \delta_{0} \leq u_{0} \\
l_{2} \leq \delta_{2} \leq u_{2}}} f\left(\mathbf{r}, p, s, \delta_{0},-\delta_{0}-\delta_{2}, \delta_{2}\right), \max _{\substack{l_{1} \leq \delta_{1} \leq u_{1} \\
l_{2} \leq \delta_{2} \leq u_{2}}} f\left(\mathbf{r}, p, s,-\delta_{1}-\delta_{2}, \delta_{1}, \delta_{2}\right)\right\} .
\end{aligned}
$$

Moreover, for any $p$ and $s$, there exist complex numbers $a, b, c, d$ such that $f\left(\mathbf{r}, p, s, \delta_{0}, \delta_{1}, \delta_{2}\right)=\left|a+b \delta_{0}+c \delta_{1}+d \delta_{2}\right|$. By Lemma 10, the maximum is then reached on a vertex. Thus, for instance for the first of these maxima we have

$\max _{\substack{l_{0} \leq \delta_{0} \leq u_{0} \\ l_{1} \leq \delta_{1} \leq u_{1}}} f\left(\mathbf{r}, p, s, \delta_{0}, \delta_{1},-\delta_{0}-\delta_{1}\right)=\max _{\left(\begin{array}{l}\delta_{0} \\ \delta_{1}\end{array}\right) \in\left\{\left(\begin{array}{l}l_{0} \\ l_{1}\end{array}\right),\left(\begin{array}{l}l_{0} \\ u_{1}\end{array}\right),\left(\begin{array}{l}u_{0} \\ l_{1}\end{array}\right),\left(\begin{array}{l}u_{0} \\ u_{1}\end{array}\right)\right\}} f\left(\mathbf{r}, p, s, \delta_{0}, \delta_{1},-\delta_{0}-\delta_{1}\right)$.

A similar conclusion can be reached for the three other maxima and we can easily compute a good upper bound on $c_{2}(\mathbf{r}, p, s)$.

The second part of the statement of the previous lemma is useful for the following result.

Lemma 24. In the statement of Lemma 23, if $\mathbf{r}$ is a left eigenvector of $M_{\tau}$ associated with an eigenvalue of modulus less than 1 , then $c_{1}(\mathbf{r}, p, s)=0$.

Proof. Using Lemma 22, we get, for all words $p \in \operatorname{Pref}\left(\tau^{n}\right)$ and $s \in \operatorname{Suff}\left(\tau^{n}\right)$,

$$
\mathbf{r} \cdot P_{3}\left(\Psi^{\prime}(p) \otimes \boldsymbol{\alpha}+\boldsymbol{\alpha} \otimes \Psi^{\prime}(s)\right)=0 .
$$

This concludes the proof. 
We can now obtain two different kinds of bounds on extended Parikh vectors of factors of the Tribonacci word. First we essentially take care of the large eigenvalues.

Proposition 25. Let $\mathbf{r}$ be a left eigenvector of $M_{\tau}$ having $\lambda$ as associated eigenvalue. If $|\lambda|<\theta$, then there exists a constant $C(\mathbf{r})$ such that, for all factors $w$ of $\mathcal{T}$,

$$
\frac{|\mathbf{r} \cdot \Psi(w)|}{|w|} \leq C(\mathbf{r}) .
$$

Proof. Let $n$ be a positive integer. We work on $\tau^{n}$ because it gives us a degree of freedom to obtain smaller bounds, but working on $\tau$ is enough to get a bound.

Let $w=w_{0}$ be a factor of $\mathcal{T}$. There exist a non-empty factor of the Tribonacci word $w_{1}, p \in \operatorname{Pref}\left(\tau^{n}(a)\right)$ and $s \in \operatorname{Suff}\left(\tau^{n}(b)\right)$, where $a$ (resp., $b$ ) is the first (resp., last) letter of $w_{1}$, such that $p w s=\tau^{n}\left(w_{1}\right)$. We say that $w_{1}$ is the preimage of $w_{0}$ (by $\left.\tau^{n}\right)$. In particular $\Psi(p w s)=\Psi\left(\tau^{n}\left(w_{1}\right)\right)$. Using the same arguments as in Lemma 7, one has

$$
\Psi(p w s)=\Psi(w)+\Psi(p s)+P_{3}\left(\Psi^{\prime}(p) \otimes \Psi^{\prime}(w)+\Psi^{\prime}(w) \otimes \Psi^{\prime}(s)\right) .
$$

Hence, we have

$$
\begin{aligned}
\Psi(w) & =\Psi\left(\tau^{n}\left(w_{1}\right)\right)-\Psi(p s)-P_{3}\left(\Psi^{\prime}(p) \otimes \Psi^{\prime}(w)+\Psi^{\prime}(w) \otimes \Psi^{\prime}(s)\right) \\
\mathbf{r} \cdot \Psi(w) & =\mathbf{r} \cdot \Psi\left(\tau^{n}\left(w_{1}\right)\right)-\mathbf{r} \cdot\left(\Psi(p s)+P_{3}\left(\Psi^{\prime}(p) \otimes \Psi^{\prime}(w)+\Psi^{\prime}(w) \otimes \Psi^{\prime}(s)\right)\right) \\
\mathbf{r} \cdot \Psi(w) & =\mathbf{r} \cdot\left(M_{\tau}^{n} \Psi\left(w_{1}\right)\right)-\mathbf{r} \cdot\left(\Psi(p s)+P_{3}\left(\Psi^{\prime}(p) \otimes \Psi^{\prime}(w)+\Psi^{\prime}(w) \otimes \Psi^{\prime}(s)\right)\right) \\
\mathbf{r} \cdot \Psi(w) & =\lambda^{n} \mathbf{r} \cdot \Psi\left(w_{1}\right)-\mathbf{r} \cdot\left(\Psi(p s)+P_{3}\left(\Psi^{\prime}(p) \otimes \Psi^{\prime}(w)+\Psi^{\prime}(w) \otimes \Psi^{\prime}(s)\right)\right) \\
|\mathbf{r} \cdot \Psi(w)| & \leq|\lambda|^{n}\left|\mathbf{r} \cdot \Psi\left(w_{1}\right)\right|+\left|\mathbf{r} \cdot\left(\Psi(p s)+P_{3}\left(\Psi^{\prime}(p) \otimes \Psi^{\prime}(w)+\Psi^{\prime}(w) \otimes \Psi^{\prime}(s)\right)\right)\right| .
\end{aligned}
$$

From Lemma 23, we get

$$
|\mathbf{r} \cdot \Psi(w)| \leq|\lambda|^{n}\left|\mathbf{r} \cdot \Psi\left(w_{1}\right)\right|+c_{1}(\mathbf{r}, p, s)|w|+c_{2}(\mathbf{r}, p, s) .
$$

Let $\ell$ be some integer with $\ell>\theta^{n}\left(2+\frac{1.5}{\theta-1}\right)$. Assume that $|w| \geq \ell$. From Corollary 20, $\left|w_{1}\right| \geq 2$. Morevover, if $w_{1}=a_{1} a_{2} \cdots a_{\left|w_{1}\right|-1} a_{\left|w_{1}\right|}$, then we can apply Corollary 20 on $a_{2} \cdots a_{\left|w_{1}\right|-1}$ :

$$
\begin{aligned}
\left|\tau^{n}\left(a_{2} a_{3} \ldots a_{\left|w_{1}\right|-1}\right)\right| & \geq \theta^{n}\left(\left|w_{1}\right|-2\right)-\theta^{n} \frac{1.5}{\theta-1} \\
|w|\left(1+\frac{\theta^{n}\left(2+\frac{1.5}{\theta-1}\right)}{|w|}\right) & \geq \theta^{n}\left|w_{1}\right| \\
|w| \frac{\ell+\theta^{n}\left(2+\frac{1.5}{\theta-1}\right)}{\ell} & \geq \theta^{n}\left|w_{1}\right| \\
|w| & \geq \theta^{n}\left|w_{1}\right| \frac{\ell}{\ell+\theta^{n}\left(2+\frac{1.5}{\theta-1}\right)} .
\end{aligned}
$$


For the sake of notation, let $\iota(\ell, n):=\frac{\ell}{\ell+\theta^{n}\left(2+\frac{1.5}{\theta-1}\right)}$. Otherwise stated, $|w| \geq$ $\theta^{n}\left|w_{1}\right| \iota(\ell, n)$. We can now compute the following bound:

$$
\begin{aligned}
& \frac{|\mathbf{r} \cdot \Psi(w)|}{|w|} \leq \frac{|\lambda|^{n}\left|\mathbf{r} \cdot \Psi\left(w_{1}\right)\right|}{|w|}+c_{1}(\mathbf{r}, p, s)+\frac{c_{2}(\mathbf{r}, p, s)}{|w|} \\
& \frac{|\mathbf{r} \cdot \Psi(w)|}{|w|} \leq \frac{|\lambda|^{n}}{\iota(\ell, n) \theta^{n}} \frac{\left|\mathbf{r} \cdot \Psi\left(w_{1}\right)\right|}{\left|w_{1}\right|}+c_{1}(\mathbf{r}, p, s)+\frac{c_{2}(\mathbf{r}, p, s)}{\ell} .
\end{aligned}
$$

Let $c_{3}(\mathbf{r})=\max \left\{c_{1}(\mathbf{r}, p, s)+\frac{c_{2}(\mathbf{r}, p, s)}{\ell}: p \in \operatorname{Pref}\left(\tau^{n}\right), s \in \operatorname{Suff}\left(\tau^{n}\right)\right\}$. We get

$$
\frac{|\mathbf{r} \cdot \Psi(w)|}{|w|} \leq \frac{|\lambda|^{n}}{\iota(\ell, n) \theta^{n}} \frac{\left|\mathbf{r} \cdot \Psi\left(w_{1}\right)\right|}{\left|w_{1}\right|}+c_{3}(\mathbf{r}) .
$$

The reader may notice that on the r.h.s., we have a factor of the same form as the 1.h.s. so, if $\left|w_{1}\right| \geq \ell$, we are tempted to apply again the same inequality on $w_{1}$. Let $w_{2}$ be the preimage of $w_{1}$ (as defined in the beginning of this proof). We can define a sequence $w=w_{0}, w_{1}, w_{2}, \ldots$ of factors of $\mathcal{T}$ such that $w_{i+1}$ is the preimage of $w_{i}$ by $\tau^{n}$.

By induction on (5), one can find a factor $w_{i}$ of $\mathcal{T}$ with $\left|w_{i}\right| \leq \ell$ such that

$$
\frac{|\mathbf{r} \cdot \Psi(w)|}{|w|} \leq\left(\frac{|\lambda|^{n}}{\iota(\ell, n) \theta^{n}}\right)^{i} \frac{\left|\mathbf{r} \cdot \Psi\left(w_{i}\right)\right|}{\left|w_{i}\right|}+c_{3}(\mathbf{r}) \sum_{k=0}^{i-1}\left(\frac{|\lambda|^{n}}{\iota(\ell, n) \theta^{n}}\right)^{k} .
$$

By assumption, $|\lambda|<\theta$, so numerically computing the modulus of the eigenvalues of $M_{\tau}$ distinct from $\theta$ and $\theta^{2}$, we obtain $|\lambda| / \theta<.74$. For all $n$, there exists a large enough $\ell$ such that $\iota(\ell, n)>.74$. Indeed for a fixed $n$, $\lim _{\ell \rightarrow+\infty} \iota(\ell, n)=1$. For such a choice, we have

$$
\frac{|\lambda|^{n}}{\iota(\ell, n) \theta^{n}} \leq \frac{|\lambda|}{\iota(\ell, n) \theta}<1
$$

Now that we have shown that a convenient pair $(\ell, n)$ exists, in practice, it is enough to choose a pair such that

$$
\frac{|\lambda|^{n}}{\iota(\ell, n) \theta^{n}}<1
$$

Since

$$
\left(\frac{|\lambda|^{n}}{\iota(\ell, n) \theta^{n}}\right)^{i}<\frac{|\lambda|^{n}}{\iota(\ell, n) \theta^{n}} \text { and } \sum_{k=0}^{i-1}\left(\frac{|\lambda|^{n}}{\iota(\ell, n) \theta^{n}}\right)^{k}<\frac{1}{1-\frac{|\lambda|^{n}}{\iota(\ell, n) \theta^{n}}},
$$

we obtain the following bound:

$$
\frac{|\mathbf{r} \cdot \Psi(w)|}{|w|} \leq \frac{|\lambda|^{n}}{\iota(\ell, n) \theta^{n}} \frac{\left|\mathbf{r} \cdot \Psi\left(w_{i}\right)\right|}{\left|w_{i}\right|}+c_{3}(\mathbf{r}) \frac{\iota(\ell, n) \theta^{n}}{\iota(\ell, n) \theta^{n}-|\lambda|^{n}} .
$$

Finally, the quantity $\frac{\left|\mathbf{r} \cdot \Psi\left(w_{i}\right)\right|}{\left|w_{i}\right|}$ is bounded by

$$
\max _{\substack{u \in \mathcal{L}(\tau) \\|u| \leq \ell}} \frac{|\mathbf{r} \cdot \Psi(u)|}{|u|}
$$


To conclude, for any positive integers $n$ and $\ell$ with

$$
\frac{|\lambda|^{n}}{\iota(\ell, n) \theta^{n}}<1 \text { and } \ell>\theta^{n}\left(2+\frac{1.5}{\theta-1}\right)
$$

and any factor $w$ of the Tribonacci word (without length restriction on $w$ ), we have

$\frac{|\mathbf{r} \cdot \Psi(w)|}{|w|} \leq \max \left\{\max _{\substack{u \in \mathcal{L}(\tau) \\|u| \leq \ell}} \frac{|\mathbf{r} \cdot \Psi(u)|}{|u|}, \frac{|\lambda|^{n}}{\iota(\ell, n) \theta^{n}} \max _{\substack{u \in \mathcal{L}(\tau) \\|u| \leq \ell}} \frac{|\mathbf{r} \cdot \Psi(u)|}{|u|}+c_{3}(\mathbf{r}) \frac{\iota(\ell, n) \theta^{n}}{\iota(\ell, n) \theta^{n}-|\lambda|^{n}}\right\}$.

As already discussed for any fixed $n$, there exists a large enough $\ell$ such that this is true. Since there are only finitely many factors of bounded size, this quantity is clearly defined and easy to compute. This concludes the proof.

Note that a good choice of $n$ and $\ell$ yields better bounds for the computations that have to be carried on. In particular, we want $\ell$ as large as possible, unfortunately $\ell$ has to be limited to the hundreds in order for the computations to be feasible. Discussions about the choice of $n$ and $\ell$ are postponed at the end of this paper.

The bound given by Proposition 25 will only be useful for the eigenvalues $\lambda$ such that $|\lambda| \geq 1$. When $|\lambda|<1$, the following result is stronger.

Proposition 26. Let $\mathbf{r}$ be an eigenvector of $M_{\tau}$ and $\lambda$ be the associated eigenvalue. If $|\lambda|<1$, then there exists a constant $C(\mathbf{r})$ such that for all factors $w$ of $\mathcal{T}$,

$$
|\mathbf{r} \cdot \Psi(w)| \leq C(\mathbf{r}) .
$$

Proof. We can reproduce the beginning of the proof of Proposition 25 and get (4). But since $|\lambda|<1$, Lemma 24 implies that $c_{1}(\mathbf{r}, p, s)=0$. So, we have

$$
|\mathbf{r} \cdot \Psi(w)| \leq|\lambda|^{n}\left|\mathbf{r} \cdot \Psi\left(w_{1}\right)\right|+c_{2}(\mathbf{r}, p, s)
$$

where $w_{1}$ is a preimage of $w$. We can iterate the process by taking preimages and we have a sequence of factors $w=w_{0}, w_{1}, w_{2}, \ldots$. We deduce by induction that for any integer $i$,

$$
|\mathbf{r} \cdot \Psi(w)| \leq|\lambda|^{n i}\left|\mathbf{r} \cdot \Psi\left(w_{i}\right)\right|+\sum_{j=0}^{i-1}|\lambda|^{j n} \max _{\substack{p \in \operatorname{Pref}\left(\tau^{n}\right) \\ s \in \operatorname{Suff}\left(\tau^{n}\right)}} c_{2}(\mathbf{r}, p, s) .
$$

Moreover, there exists an integer $k$, such that for all $i \geq k,\left|w_{i}\right|=1$. Then, for all $i \geq k$,

$$
|\mathbf{r} \cdot \Psi(w)| \leq|\lambda|^{n i} \max _{a \in \Sigma}|\mathbf{r} \cdot \Psi(a)|+\sum_{j=0}^{i-1}|\lambda|^{j n} \max _{\substack{p \in \operatorname{Pref}\left(\tau^{n}\right) \\ s \in \operatorname{Suff}\left(\tau^{n}\right)}} c_{2}(\mathbf{r}, p, s) .
$$


This implies

$$
\begin{aligned}
& |\mathbf{r} \cdot \Psi(w)| \leq \lim _{i \rightarrow \infty}\left(|\lambda|^{n i} \max _{a \in \Sigma}|\mathbf{r} \cdot \Psi(a)|+\sum_{j=0}^{i-1}|\lambda|^{j n} \max _{\substack{p \in \operatorname{Pref}\left(\tau^{n}\right) \\
s \in \operatorname{Suff}\left(\tau^{n}\right)}} c_{2}(\mathbf{r}, p, s)\right) \\
& |\mathbf{r} \cdot \Psi(w)| \leq \max _{\substack{p \in \operatorname{Pref}\left(\tau^{n}\right) \\
s \in \operatorname{Suff}\left(\tau^{n}\right)}} \frac{c_{2}(\mathbf{r}, p, s)}{1-|\lambda|^{n}}
\end{aligned}
$$

and concludes the proof.

4.2. Bounds on templates. This subsection contains several lemmas giving necessary conditions on templates to be realizable by $\tau$.

Lemma 27. Let $\lambda$ be an eigenvalue of $M_{\tau}$ such that $|\lambda|<1$. For every left eigenvector $\mathbf{r}$ of $M_{\tau}$ associated with $\lambda$ and for every realizable template $t=\left[\mathbf{d}, \mathbf{D}_{\mathbf{b}}, \mathbf{D}_{\mathbf{e}}, a_{1}, a_{2}\right]$,

$\min _{\left(\delta_{0}, \delta_{1}\right) \in \Delta}\left|\mathbf{r} \cdot\left(\mathbf{d}+P_{3}\left(\mathbf{D}_{\mathbf{b}} \otimes\left(\begin{array}{c}\delta_{0} \\ \delta_{1} \\ -\delta_{0}-\delta_{1}\end{array}\right)+\left(\begin{array}{c}\delta_{0} \\ \delta_{1} \\ -\delta_{0}-\delta_{1}\end{array}\right) \otimes \mathbf{D}_{\mathbf{e}}\right)\right)\right| \leq 2 C(\mathbf{r})$

where $C(\mathbf{r})$ is the constant from Proposition 26.

Proof. From Proposition 26, for any two factors $u, v \in \mathcal{L}(\tau)$, we have $|\mathbf{r} \cdot(\Psi(u)-\Psi(v))| \leq 2 C(\mathbf{r})$. On the other hand, $t$ is realizable so there are two factors $u$ and $v$ of the Tribonacci word such that

$$
\Psi(u)-\Psi(v)=\mathbf{d}+P_{3}\left(\mathbf{D}_{\mathbf{b}} \otimes \Psi^{\prime}(u)+\Psi^{\prime}(u) \otimes \mathbf{D}_{\mathbf{e}}\right) .
$$

This implies

$$
\left|\mathbf{r} \cdot\left(\mathbf{d}+P_{3}\left(\mathbf{D}_{\mathbf{b}} \otimes \Psi^{\prime}(u)+\Psi^{\prime}(u) \otimes \mathbf{D}_{\mathbf{e}}\right)\right)\right| \leq 2 C(\mathbf{r}) .
$$

From Corollary 18 , there are $\left(\delta_{0}, \delta_{1}\right) \in \Delta$ such that

$$
\Psi^{\prime}(w)=|w|\left(\begin{array}{c}
\alpha_{0} \\
\alpha_{1} \\
\alpha_{2}
\end{array}\right)+\left(\begin{array}{c}
\delta_{0} \\
\delta_{1} \\
-\delta_{0}-\delta_{1}
\end{array}\right)
$$

We write again $\boldsymbol{\alpha}=\left(\begin{array}{lll}\alpha_{0} & \alpha_{1} & \alpha_{2}\end{array}\right)^{\top}$ and $\boldsymbol{\delta}=\left(\begin{array}{lll}\delta_{0} & \delta_{1} & -\delta_{0}-\delta_{1}\end{array}\right)^{\top}$.

It gives

$$
2 C(\mathbf{r}) \geq\left|\mathbf{r} \cdot\left(\mathbf{d}+P_{3}\left(\mathbf{D}_{\mathbf{b}} \otimes(\boldsymbol{\alpha}|u|+\boldsymbol{\delta})+(\boldsymbol{\alpha}|u|+\boldsymbol{\delta}) \otimes \mathbf{D}_{\mathbf{e}}\right)\right)\right| .
$$

From Lemma 22, we get

$$
\mathbf{r} \cdot P_{3}\left(\mathbf{D}_{\mathbf{b}} \otimes \boldsymbol{\alpha}|u|\right)=\mathbf{r} \cdot P_{3}\left(\boldsymbol{\alpha}|u| \otimes \mathbf{D}_{\mathbf{e}}\right)=0
$$

and thus we finally obtain

$$
\begin{aligned}
2 C(\mathbf{r}) & \geq\left|\mathbf{r} \cdot\left(\mathbf{d}+P_{3}\left(\mathbf{D}_{\mathbf{b}} \otimes \boldsymbol{\delta}+\boldsymbol{\delta} \otimes \mathbf{D}_{\mathbf{e}}\right)\right)\right| \\
& \geq \min _{\left(\delta_{0}, \delta_{1}\right) \in \Delta}\left|\mathbf{r} \cdot\left(\mathbf{d}+P_{3}\left(\mathbf{D}_{\mathbf{b}} \otimes\left(\begin{array}{c}
\delta_{0} \\
\delta_{1} \\
-\delta_{0}-\delta_{1}
\end{array}\right)+\left(\begin{array}{c}
\delta_{0} \\
\delta_{1} \\
-\delta_{0}-\delta_{1}
\end{array}\right) \otimes \mathbf{D}_{\mathbf{e}}\right)\right)\right|
\end{aligned}
$$


This bound is not so easy to use because of the complicated minimum. It can be computed using tools from optimization. However, we can simply use this bound as follows.

First, for the sake of notation, let

$$
f\left(\delta_{0}, \delta_{1}\right)=\mathbf{r} \cdot\left(\mathbf{d}+P_{3}\left(\mathbf{D}_{\mathbf{b}} \otimes\left(\begin{array}{c}
\delta_{0} \\
\delta_{1} \\
-\delta_{0}-\delta_{1}
\end{array}\right)+\left(\begin{array}{c}
\delta_{0} \\
\delta_{1} \\
-\delta_{0}-\delta_{1}
\end{array}\right) \otimes \mathbf{D}_{\mathbf{e}}\right)\right) .
$$

Then

$$
\min _{\left(\delta_{0}, \delta_{1}\right) \in \Delta}\left|f\left(\delta_{0}, \delta_{1}\right)\right| \geq \sqrt{\min _{\left(\delta_{0}, \delta_{1}\right) \in \Delta} \operatorname{Re}\left(f\left(\delta_{0}, \delta_{1}\right)\right)^{2}+\min _{\left(\delta_{0}, \delta_{1}\right) \in \Delta} \operatorname{Im}\left(f\left(\delta_{0}, \delta_{1}\right)\right)^{2}} .
$$

Let $I_{R e}$ and $I_{I m}$ be intervals such that

$$
I_{R e}=\left[\min _{\left(\delta_{0}, \delta_{1}\right) \in \Delta} \operatorname{Re}\left(f\left(\delta_{0}, \delta_{1}\right)\right), \max _{\left(\delta_{0}, \delta_{1}\right) \in \Delta} \operatorname{Re}\left(f\left(\delta_{0}, \delta_{1}\right)\right)\right]
$$

and

$$
I_{I m}=\left[\min _{\left(\delta_{0}, \delta_{1}\right) \in \Delta} \operatorname{Im}\left(f\left(\delta_{0}, \delta_{1}\right)\right), \max _{\left(\delta_{0}, \delta_{1}\right) \in \Delta} \operatorname{Im}\left(f\left(\delta_{0}, \delta_{1}\right)\right)\right] .
$$

Then

$$
\min _{\left(\delta_{0}, \delta_{1}\right) \in \Delta}\left|f\left(\delta_{0}, \delta_{1}\right)\right| \geq \sqrt{\min _{y \in I_{R e}} y^{2}+\min _{y \in I_{I m}} y^{2}} .
$$

Thus any template for which this last quantity is greater than $2 C(\mathbf{r})$ is not realizable.

Observe that each of the four interval bounds is reached for a vertex of the polytope, that is $\left(\begin{array}{c}\delta_{0} \\ \delta_{1}\end{array}\right) \in\left\{\left(\begin{array}{c}1.5 \\ -1.5\end{array}\right),\left(\begin{array}{c}1.5 \\ 0\end{array}\right),\left(\begin{array}{c}0 \\ 1.5\end{array}\right),\left(\begin{array}{c}-1.5 \\ 1.5\end{array}\right),\left(\begin{array}{c}-1.5 \\ 0\end{array}\right),\left(\begin{array}{c}0 \\ -1.5\end{array}\right)\right\}$. This is due to the fact that $f$ is linear (and thus convex) over the convex set $\Delta$. The proof of this fact is identical to the proof of Lemma 10 .

This allows us to remove many templates from the set of templates, but this is not enough to have a finite set so we need to somehow use the bounds on the other eigenvectors as well.

Lemma 28. Let $L$ be a positive integer. Let $\lambda$ be an eigenvalue of $M_{\tau}$ such that $|\lambda|<\theta$. Then, for all eigenvectors $\mathbf{r}$ of $M_{\tau}$ associated with $\lambda$, there exists a constant $C(\mathbf{r})$ such that for any template $t=\left[\mathbf{d}, \mathbf{D}_{\mathbf{b}}, \mathbf{D}_{\mathbf{e}}, a_{1}, a_{2}\right]$ realized by a pair of factors of the Tribonacci word $(u, v)$ with $|u| \geq L$, we have

$$
\begin{aligned}
& \left|\mathbf{r} \cdot P_{3}\left(\mathbf{D}_{\mathbf{b}} \otimes \boldsymbol{\alpha}+\boldsymbol{\alpha} \otimes \mathbf{D}_{\mathbf{e}}\right)\right| \leq \\
& \quad \frac{2 L-\sum_{i=1}^{3} \mathbf{d}_{i}}{L} C(\mathbf{r})+\max _{\left(\delta_{0}, \delta_{1}\right) \in \Delta} \frac{\left|\mathbf{r} \cdot\left(\mathbf{d}+P_{3}\left(\mathbf{D}_{\mathbf{b}} \otimes \boldsymbol{\delta}+\boldsymbol{\delta} \otimes \mathbf{D}_{\mathbf{e}}\right)\right)\right|}{L} .
\end{aligned}
$$

Proof. From Proposition 25, there is a constant $C(\mathbf{r})$ such that for any two factors $u, v \in \mathcal{L}(\tau)$, we have $|\mathbf{r} \cdot(\Psi(u)-\Psi(v))| \leq(|u|+|v|) C(\mathbf{r})$. On the 
other hand, $t$ is realized by a pair of factors $(u, v)$ of $\mathcal{T}$ with $|u| \geq L$ such that

$$
\Psi(u)-\Psi(v)=\mathbf{d}+P_{3}\left(\mathbf{D}_{\mathbf{b}} \otimes \Psi^{\prime}(u)+\Psi^{\prime}(u) \otimes \mathbf{D}_{\mathbf{e}}\right) .
$$

This implies

$$
\left|\mathbf{r} \cdot\left(\mathbf{d}+P_{3}\left(\mathbf{D}_{\mathbf{b}} \otimes \Psi^{\prime}(u)+\Psi^{\prime}(u) \otimes \mathbf{D}_{\mathbf{e}}\right)\right)\right| \leq(|u|+|v|) C(\mathbf{r}) .
$$

From Corollary 18 , there is $\left(\delta_{0}, \delta_{1}\right) \in \Delta$ such that

$$
\Psi^{\prime}(w)=|w|\left(\begin{array}{l}
\alpha_{0} \\
\alpha_{1} \\
\alpha_{2}
\end{array}\right)+\left(\begin{array}{c}
\delta_{0} \\
\delta_{1} \\
-\delta_{0}-\delta_{1}
\end{array}\right) .
$$

We write again $\boldsymbol{\alpha}=\left(\begin{array}{lll}\alpha_{0} & \alpha_{1} & \alpha_{2}\end{array}\right)^{\top}$ and $\boldsymbol{\delta}=\left(\begin{array}{lll}\delta_{0} & \delta_{1} & -\delta_{0}-\delta_{1}\end{array}\right)^{\top}$.

It gives

$$
(|u|+|v|) C(\mathbf{r}) \geq\left|\mathbf{r} \cdot\left(\mathbf{d}+P_{3}\left(\mathbf{D}_{\mathbf{b}} \otimes(\boldsymbol{\alpha}|u|+\boldsymbol{\delta})+(\boldsymbol{\alpha}|u|+\boldsymbol{\delta}) \otimes \mathbf{D}_{\mathbf{e}}\right)\right)\right| .
$$

We can now apply the triangular inequality and divide each side by $|u|$ :

$$
\left|\mathbf{r} \cdot P_{3}\left(\mathbf{D}_{\mathbf{b}} \otimes \boldsymbol{\alpha}+\boldsymbol{\alpha} \otimes \mathbf{D}_{\mathbf{e}}\right)\right| \leq \frac{|u|+|v|}{|u|} C(\mathbf{r})+\frac{\left|\mathbf{r} \cdot\left(\mathbf{d}+P_{3}\left(\mathbf{D}_{\mathbf{b}} \otimes \boldsymbol{\delta}+\boldsymbol{\delta} \otimes \mathbf{D}_{\mathbf{e}}\right)\right)\right|}{|u|} .
$$

Finally, we can use the inequality $|u| \geq L$ :

$$
\begin{aligned}
& \left|\mathbf{r} \cdot P_{3}\left(\mathbf{D}_{\mathbf{b}} \otimes \boldsymbol{\alpha}+\boldsymbol{\alpha} \otimes \mathbf{D}_{\mathbf{e}}\right)\right| \leq \\
& \frac{2 L-\sum_{i=1}^{3} \mathbf{d}_{i}}{L} C(\mathbf{r})+\max _{\left(\delta_{0}, \delta_{1}\right) \in \Delta} \frac{\left|\mathbf{r} \cdot\left(\mathbf{d}+P_{3}\left(\mathbf{D}_{\mathbf{b}} \otimes \boldsymbol{\delta}+\boldsymbol{\delta} \otimes \mathbf{D}_{\mathbf{e}}\right)\right)\right|}{L} .
\end{aligned}
$$

This concludes the proof.

The quantity of the 1.h.s. and the first term on the r.h.s. in (8) are straightforward to compute. For the last term, Lemma 10 tells us that the maximum is in fact necessarily reached on a vertex of the polytope, that is

$$
\begin{aligned}
& \max _{\left(\delta_{0}, \delta_{1}\right) \in \Delta} \frac{\left|\mathbf{r} \cdot\left(\mathbf{d}+P_{3}\left(\mathbf{D}_{\mathbf{b}} \otimes \boldsymbol{\delta}+\boldsymbol{\delta} \otimes \mathbf{D}_{\mathbf{e}}\right)\right)\right|}{L} \leq \\
& \left(\begin{array}{c}
\delta_{0} \\
\delta_{1}
\end{array}\right) \in\left\{\left(\begin{array}{c}
1.5 \\
0
\end{array}\right),\left(\begin{array}{c}
1.5 \\
-1.5
\end{array}\right),\left(\begin{array}{c}
0 \\
1.5
\end{array}\right),\left(\begin{array}{c}
-1.5 \\
0
\end{array}\right),\left(\begin{array}{c}
-1.5 \\
1.5
\end{array}\right),\left(\begin{array}{c}
0 \\
-1.5
\end{array}\right)\right\}
\end{aligned}
$$

\section{Proof of the main Result}

With all these lemmas, we are ready to show our main result.

Theorem 29. Two factors of the Tribonacci word are 2-binomially equivalent if and only if they are equal.

Proof. Let $T=\left\{\left[\mathbf{0}, \mathbf{0}, \mathbf{0}, a_{1}, a_{2}\right]: a_{1} \neq a_{2}\right\}$. Let us show that no template from $T$ is realizable. Let $L=15$. We can easily check with a computer that no pair of factors of $\mathcal{T}$ with $\min (|u|,|v|) \leq L$ realizes a template $t$ from the set $T$. Indeed, since for all $t \in T, \mathbf{d}=\mathbf{0}, \mathbf{D}_{\mathbf{b}}=\mathbf{0}$ and $\mathbf{D}_{\mathbf{e}}=\mathbf{0}$, we know 
that a pair of words $(u, v)$ realizes $t$ if and only if $\Psi(u)-\Psi(v)=0$. It just suffices to check that for all $n \leq L, b_{\mathcal{T}, 2}(n)=p_{\mathcal{T}}(n)$.

Now, from Proposition 17, if $t \in T$ is realized then one of its ancestors is realized by a pair $(u, v)$ with $L \leq \min (|u|,|v|) \leq 2 L$.

Lemmas 27 and 28 give us two sets of inequalities that any template realized by a pair $(u, v)$ of factors of Tribonacci with $|u| \geq L$ must respect. Let $X$ be the set of templates that respect the bounds. Let $A_{0}=T$ and, for all $i$, let $A_{i+1}=\left\{\operatorname{Par}_{\tau}(t) \cap X: t \in A_{i}\right\}$. Then clearly $\operatorname{RAnc}_{\tau}(t) \subseteq \bigcup_{i \in \mathbb{N}} A_{i}$.

Each $A_{i}$ can be easily computed and it can be checked by a computer program that the set $\bigcup_{i \in \mathbb{N}} A_{i}$ is finite.

We can finally check with a computer that there is no pair $(u, v)$ of factors of $\mathcal{T}$ with $L \leq \min (|u|,|v|) \leq 2 L$ that realizes any element of $\bigcup_{i \in \mathbb{N}} A_{i}$. Thus no template of $T$ is realizable. By Lemma 13, we can conclude that the 2-binomial complexity of the Tribonacci word is equal to its factorial complexity.

We provide with this paper an implementation in Mathematica of all the computations described in this theorem and in the previous lemmas and propositions. We also have a $\mathrm{C}++$ implementation that is much faster, but uses machine floating point arithmetic whose accuracy cannot be guaranted (in this case however we obtain exactly the same set of templates). Diagonalizing the matrix of Tribonacci gives 4 eigenvectors to which Lemma 27 can be applied. Since there are two pairs of conjugate complex vectors, it is useless to keep more than one of each pair. However, by taking a linear combination of these two, we get another eigenvector to which we can apply Lemma 27 (in practice we only do that once, but we could take as many vectors as we want from this 2-dimensional space). For this conjugation reason, we also only keep 4 of the 6 eigenvectors that correspond to an eigenvalue of norm less than 1 . For each of these 7 eigenvectors, we choose ${ }^{2} \ell=600$ and the best $1 \leq n \leq 6$ when applying Lemma 27 or Lemma 28 . The rest is done as described in the article. We obtain a set of 241544 templates.

\section{Conclusion}

We used an algorithm to show that the 2-binomial complexity of the Tribonacci word is equal to its factorial complexity. It seems that our method can be turned into an algorithm that can decide under some mild conditions whether the factorial complexity of a given morphic word is equal to its $k$-binomial complexity. In fact, by keeping track of the first letter of each word in templates, the "if" in Proposition 17 can be replaced by an "if and only if" (some technicalities could allow us to apply it even if the matrix is singular). Moreover, with arguments similar to the ideas from [15], one

\footnotetext{
${ }^{2}$ Remember that we work on $\tau^{n}$ and that increasing $n$ and $\ell$ tend to give us better bounds but increases the computation time.
} 
could show that we also have bounds on the eigenvectors that correspond to larger eigenvalues and that the number of templates that respect these bounds is always finite (one might need that no eigenvalues has norm 1).

Observe that the notion of template was first introduced in the context of avoidability of abelian powers [8] and, as one could expect, it seems that our technique also gives a decision algorithm for the avoidability of $k$-binomial powers in morphic words (and even avoidability of patterns in the $k$-binomial sense).

\section{REFERENCES}

[1] A. Aberkane, J. Currie, A cyclic binary morphism avoiding abelian fourth powers, Theoret. Comput. Sci. 410 (2009), 44-52.

[2] A. Aberkane, J. Currie, N. Rampersad, The number of ternary words avoiding abelian cubes grows exponentially, J. Integer Seq. 7 (2004), Article 04.2.7.

[3] J.-P. Allouche, J. Shallit, Automatic sequences. Theory, applications, generalizations, Cambridge University Press (2003).

[4] V. Berthé, M. Rigo (Eds.), Combinatorics, Automata and Number Theory, Encyclopedia Math. Appl., 135, Cambridge Univ. Press (2010).

[5] J. Cassaigne, J. Currie, L. Schaeffer, J. Shallit, Avoiding three consecutive blocks of the same size and same sum, J. ACM 61 (2014), no. 2, Art. 10.

[6] J. Cassaigne, G. Richomme, K. Saari, L.Q. Zamboni, Avoiding Abelian powers in binary words with bounded Abelian complexity, Internat. J. Found. Comput. Sci. 22 (2011), 905-920.

[7] J. Currie, N. Rampersad, Recurrent words with constant Abelian complexity, Adv. Appl. Math. 47 (2011), 116-124.

[8] J. Currie, N. Rampersad, Fixed points avoiding Abelian $k$-powers, J. Combin. Theory Ser. A 119 (2012), 942-948.

[9] A. Graham, Kronecker products and matrix calculus: with applications, Ellis Horwood Series in Mathematics and its Applications, Halsted Press [John Wiley \& Sons, Inc., New York, (1981).

[10] J. Karhumäki, A. Saarela, L. Q. Zamboni, On a generalization of Abelian equivalence and complexity of infinite words, J. Combin. Theory Ser. A 120 (2013), 2189-2206.

[11] J. Karhumäki, A. Saarela, L. Q. Zamboni, Variations of the Morse-Hedlund theorem for $k$-abelian equivalence, Lect. Notes in Comput. Sci. 8633 (2014), 203-214.

[12] M. Lejeune, J. Leroy, M. Rigo, Computing the $k$-binomial complexity of the ThueMorse word, arXiv:1812.07330, 34 pages.

[13] F. Liétard, avoiding additive powers, talk at Mons TCS days, Bordeaux 10-14 september 2018.

[14] M. Rao, M. Rigo, P. Salimov, Avoiding 2-binomial squares and cubes, Theoret. Comput. Sci. 572 (2015), 83-91.

[15] M. Rao and M. Rosenfeld, Avoiding Two Consecutive Blocks of Same Size and Same Sum over $\mathbb{Z}^{2}$, SIAM Journal on Disc. Math. 32:4 (2018), 2381-2397

[16] G. Richomme, K. Saari, L.Q. Zamboni, Balance and Abelian complexity of the Tribonacci word, Adv. Appl. Math. 45 (2010), 212-231.

[17] M. Rigo, P. Salimov, Another generalization of abelian equivalence: binomial complexity of infinite words, Theoret. Comput. Sci. 601 (2015), 47-57.

[18] M. Rigo, invited talk, Streams II, Lorentz Center, Leiden, January 2014.

[19] M. Rigo, Relations on words, Indag. Math. (N.S.) 28 (2017), 183-204.

Email address: \{M.Lejeune,M.Rigo, Matthieu.Rosenfeld\}@uliege.be 
University of Liège, Dept. of Mathematics, Allée de la découverte 12 (B37), B-4000 LiÈge, Belgium 